\title{
Movilidad urbana y personas mayores en Santiago de Chile: el valor de integrar métodos de análisis, un estudio en el barrio San Eugenio
}

\section{Urban mobility and elderlies in Santiago de Chile: The value of integrating analytical methods, a case study of the San Eugenio neighbourhood}

Giovanni Vecchio

ORCID: https://orcid.org/0000-0002-5920-1507

Instituto de Estudios Urbanos y Territoriales, Pontificia Universidad Católica de Chile, Santiago, Chile, Centro de Desarrollo Sustentable (CEDEUS), Pontificia Universidad Católica de Chile, Santiago, Chile. Correo electrónico: giovanni.vecchio@uc.cl

Bryan Castillo

ORCID: https://orcid.org/0000-0001-6793-9053

Centro de Desarrollo Sustentable (CEDEUS), Pontificia Universidad Católica de Chile, Santiago, Chile.

\author{
Stefan Steiniger \\ ORCID: https://orcid.org/0000-0002-0025-9878 \\ Centro de Desarrollo Sustentable (CEDEUS), Pontificia Universidad Católica de Chile, Santiago, Chile.
}

Esta investigación recibió el apoyo de CEDEUS - Centro de Desarrollo Urbano Sustentable (ANID/Fondap/15110020). Agradecemos la Unidad de Política y Práctica de CEDEUS por el apoyo en todas las etapas del trabajo en el barrio San Eugenio, desde el diseño de la encuesta hasta el análisis de los resultados.

\begin{abstract}
Resumen
La evolución demográfica de América Latina, y especialmente Chile, muestra un creciente envejecimiento de la población: ya en el 2050, uno de cada cuatro latinoamericanos y uno de cada tres chilenos tendrán más de 60 años. Entre los elementos que contribuyen al bienestar de las personas mayores, las posibilidades de movilidad y acceso juegan un rol fundamental y debieran entonces constituirse como prioridad en la formulación de planes y políticas de movilidad. Sin embargo, las tradicionales metodologías de la planificación de trasporte no logran capturar adecuadamente las formas en que las personas mayores se mueven y logran acceder a oportunidades urbanas. El artículo discute el valor de integrar diferentes métodos de análisis para una mejor representación de la movilidad de las personas mayores. Analizando la movilidad y la accesibilidad de una comunidad de personas mayores en el barrio San Eugenio, en Santiago de Chile, el artículo compara los resultados de entrevistas y encuestas por hogares, observando en qué medida contribuyen a la descripción de las prácticas de movilidad y acceso de la población examinada. Los resultados muestran la relevancia de enfoques cualitativos que, a escala barrial, examinan específicamente la población mayor, considerando que estos métodos hacen emerger prácticas de movilidad y patrones de accesibilidad difíciles de observar a través de análisis más agregados. La aproximación a la movilidad de las personas mayores permitida por las herramientas cualitativas requiere considerar las condiciones de su adopción por parte de la planificación de la movilidad al nivel barrial y urbano.
\end{abstract}

Palabras clave

accesibilidad, movilidad cotidiana, personas mayores, planificación del transporte.

\begin{abstract}
Demographic changes in Latin America, and especially in Chile, indicate that the population is becoming older: already in 2050, one out of four Latin Americans and one out of three Chileans will be older than 60 . Among the elements that contribute to the wellbeing of the elderlies, mobility and accessibility play a fundamental role. Priorities set out for mobility planning and politics should reflect these aspects. Nonetheless, the traditional methods of transport planning in Chile are not able to adequately grasp the forms in which elderlies move and access urban opportunities. We discuss the value of integrating different methods of analysis to better represent the mobility of elderlies. Examining the mobility and accessibility behaviour of seniors in the neighbourhood of San Eugenio, in Santiago de Chile, the paper compares the results of interviews and household travel surveys, observing to what extent these contribute to the description of mobility and accessibility for the examined population. The results show the relevance of qualitative approaches that, at the neighbourhood scale, examine elderlies, considering that these methods detect mobility practices and accessibility patterns difficult to observe with more aggregate forms of analysis. The approach to elderly mobility allowed by qualitative tools allows considering the conditions for their adoption when planning mobility at neighbourhood and municipal scale.
\end{abstract}

\section{Keywords}

accessibility, elderlies, everyday mobility, transport planning.
HISTORIAL DEL ARTÍCULO

Recibido:

23 de abril de 2020.

Aceptado:

30 de octubre de 2020. 


\section{Introducción}

La evolución demográfica de América Latina, y de Chile especialmente, muestra un creciente envejecimiento de la población, que en las próximas décadas verá un mayor número de personas mayores, y un mayor impacto en términos porcentuales sobre la sociedad. En América Latina y el Caribe, el porcentaje de adultos mayores -aquí entendidos como todas aquellas personas mayores de 60 años- tendrá un crecimiento notable: en el 2000 eran el 8,3\% de la población de la región y se estima que en el 2020 alcanzarán el 13\%; en el 2050 llegarán a ser un cuarto de los habitantes (24,9\%); y al final del siglo, las personas mayores serán el 37,9\% de la población de la región (United Nations, 2017). En Chile la dinámica de envejecimiento es más acentuada: hoy en día el país ya cuenta con 3 millones de ciudadanos sobre 60 años y las previsiones demográficas muestran que la población chilena envejecerá aún más en los próximos años, debidos a una mayor longevidad y a menores tasas de nacimiento que llevarán este grupo a ser un tercio de la población chilena ya en el 2050 (Instituto Nacional de Estadísticas, 2017; United Nations, 2017).

En este contexto, la movilidad de las personas mayores emerge como un tema prioritario para la investigación y las políticas públicas, así como demuestra la creciente atención que países de Europa y Norteamérica dedican al tema. Para las personas de tercera edad, la movilidad es un elemento fundamental que puede contribuir o afectar la autonomía y el bienestar individual (Banister \& Bowling, 2004; Goins et al., 2015; Nordbakke, 2013; Nordbakke \& Schwanen, 2014; Schwanen, Banister, \& Bowling, 2012; Stjernborg, Wretstrand \& Tesfahuney, 2015; Umstattd Meyer, Janke \& Beaujean, 2014; Webber, Porter $\&$ Menec, 2010). Por un lado, moverse es fundamental para acceder a oportunidades urbanas, es decir, aquellos lugares requeridos para satisfacer necesidades y deseos personales. Por otro lado, la posibilidad de moverse es afectada por barreras económicas, físicas y cognitivas, que tienden a aumentar en la última etapa de la vida. Por lo tanto, las posibilidades de acceso de las personas mayores son fundamentales para su bienestar, y debieran constituirse como prioridad en la formulación de planes y políticas de movilidad en el contexto de un país que se encuentra envejeciendo (Akhavan \& Vecchio, 2018; Martens, 2018).

Sin embargo, las tradicionales metodologías de la planificación de transporte no logran capturar adecuadamente las formas en que las y los adultos mayores se mueven y acceden a oportunidades. Se trata de una limitación evidenciada por el creciente grupo de trabajos enfocados en las implicancias sociales de la movilidad urbana. Tradicionalmente, la planificación del transporte se basa en disciplinas como la economía e ingeniería, con un enfoque utilitarista (Schwanen, 2018). Enfatizando en la demanda de movilidad y la eficiencia interna de los sistemas de transporte, los enfoques tradicionales de la planificación han desarrollado herramientas de modelación refinadas para predecir los flujos de personas y, consecuentemente, para poder definir las acciones necesarias para su movimiento. Estas mismas herramientas han demostrado ser limitadas para considerar los impactos sociales del transporte (Martens, 2006; Wee, 2011). Por ejemplo, este es el caso de las Encuestas Origen-Destino (en adelante, EOD) que, reconstruyendo a una escala macro los flujos de movilidad en un cierto contexto, no consideran elementos relevantes para entender la movilidad urbana y sus impactos sobre las personas. Las EOD tienden a (i) invisibilizar las formas de movilidad que no caben entre los flujos principales, (ii) asumen una perspectiva exclusivamente individual -ignorando eventuales formas de interdependencia entre personas, (iii) no logran capturar formas obligadas o voluntarias de inmovilidades decir, personas que necesitarían desplazarse, pero no tienen los recursos necesarios para hacerlo, y (iv) no consideran prácticas o experiencias de movilidad. Estas limitaciones son especialmente problemáticas en el caso de las personas mayores, cuya posibilidad de moverse y acceder a oportunidades urbanas tiene que ver con muchas dimensiones que las EOD actualmente no logran representar.

Considerando estos elementos, el trabajo pretende discutir el valor de integrar diferentes métodos de análisis para una mejor representación de la movilidad de las 
personas mayores. Para este objetivo, el trabajo analiza la movilidad y la accesibilidad de personas mayores en un barrio de la ciudad de Santiago de Chile, investigando el barrio San Eugenio (en la comuna de Santiago). El análisis consiste en la descripción y posterior caracterización de las necesidades de movilidad y acceso de este segmento de la población, basándose en (1) los resultados de la EOD para la ciudad de Santiago, (2) en una encuesta de movilidad por hogares enfocada en el barrio, y (3) en una serie de entrevistas semiestructuradas con personas mayores residentes en el barrio. Esto con el fin de observar en qué manera diferentes métodos de análisis pueden contribuir a la definición de prioridades para mejoras de infraestructura y servicios de transporte que garanticen el acceso a actividades diarias tales como ferias, negocios, centros de salud, servicios municipales, plazas y parques.

La movilidad de las personas mayores es un tema emergente que levanta desafíos específicos para Chile, como demuestra el reciente interés académico en el tema (sección 2 de este artículo). Por esto, requiere el uso de diferentes metodologías, que se complementen entre ellas y permitan el levantamiento de información tanto cuantitativa como cualitativa (sección 3 de este artículo). Describiendo oportunidades, barreras y estrategias de movilidad y acceso, los resultados del trabajo muestran la relevancia de un enfoque en la movilidad de las personas mayores y de una comparación con otras formas de análisis más agregadas (sección 4 de este artículo). De esta forma es posible, de nuestro punto de vista, reconocer las necesidades específicas de acceso de las personas mayores, así como experiencias y prácticas de movilidad requeridas para alcanzar destinos de interés para este grupo. Aunque el trabajo es una primera aproximación al tema de la movilidad y de la accesibilidad de las personas mayores en Chile, sus resultados son relevantes en tres sentidos (sección 5 y 6 de este artículo): ofrecen elementos para planes y políticas de movilidad a diferentes escalas territoriales; permiten formular hipótesis en base a la información levantada sobre las maneras en que las personas mayores construyen y hacen uso de su movilidad; ponen a prueba diferentes herramientas analíticas, mostrando el valor de integrar distintos métodos de análisis a la escala barrial. El artículo se concluye con unas preguntas abiertas sobre la relevancia de planificar la ciudad y su movilidad con un enfoque en las personas mayores, y la necesidad de fortalecer la atención al tema tanto en la investigación académica como en la opinión pública.

\section{Movilidad y personas mayores en Chile: retos de un tema emergente}

La movilidad de las personas mayores está ganando atención en la investigación a nivel internacional, debido al creciente envejecimiento de la población (sobre todo en los países del Norte Global) y a los nuevos desafíos urbanos que levanta (OECD, 2015). La posibilidad de moverse es uno de los principales elementos que contribuye al bienestar de las personas mayores, dado que permite acceder a oportunidades locales (como comprar comida, recibir atención médica, o visitar parques), participar en actividades sociales, y en general proveer para uno mismo en una edad usualmente caracterizada por reducidos niveles de autonomía (Banister \& Bowling, 2004). E1 creciente envejecimiento de la población chilena hace que el tema sea relevante también para el país, aunque la creciente investigación sobre el tema y las nuevas metodologías que esta aplica encuentran poco espacio en la planificación urbana y de la movilidad.

La movilidad de las personas mayores en Chile ha sido objeto de un número creciente de trabajos, que dependiendo de la perspectiva disciplinaria adoptada utilizan enfoques diferentes. La movilidad como habilidad básica, que permite el cumplimiento de actividades básicas y aporta al bienestar de la persona, es el elemento central de investigaciones del área médica: estas por ejemplo se enfocan en actividades básicas, como caminar una calle o subir un piso por las escaleras (Fuentes-García, Sánchez, Lera, Cea y Albala, 2013); en la capacidad de utilizar autónomamente el transporte público, también desarrollando estrategias adaptativas (Gajardo et al., 2012); y en la posibilidad de acceder a servicios de salud, sujeto a la condición socioeconómica de la persona mayor (Wallace \& Gutiérrez, 2005). La desigualdad aparece como elemento recurrente para explicar los distintos niveles 
de autonomía a disposición de las personas mayores, demostrando una vez más el impacto que la desigualdad genera en la salud en los contextos urbanos de América Latina (Bilal et al., 2019).

Desde la perspectiva de los estudios urbanos, la movilidad de las personas mayores emerge como resultado de la interacción entre la condición personal del anciano y las condiciones objetivas de los lugares que habita (por ejemplo, en términos de servicios a disposición y calidad de los espacios públicos). En comparación con otros grupos demográficos, los patrones de movilidad de las personas mayores en Santiago muestran una clara tendencia a desplazarse cada vez menos, sobre todo en el caso de personas jubiladas (Delaunay, Fournier y Contreras, 2013). Emerge un fuerte anclaje al barrio en que se reside (Lazo y Calderón, 2014) y, probablemente debido a esta dominancia de la escala barrial, la caminata se vuelve el modo de desplazamiento dominante en la tercera edad, tanto para hombres y mujeres (Figueroa Martínez \& Waintrub Santibáñez, 2015; Herrmann-Lunecke, Mora, \& Sagaris 2020). Además, las condiciones del entorno construido a menudo no permiten desplazamientos seguros, sea por el riesgo de accidentes (por ejemplo, caídas) o por la exposición a potenciales peligros, como en el caso de zonas con presencia de criminalidad. Como resultado, se observan diferentes estrategias de movilidad, "resultado de la negociación entre los recursos individuales y las condiciones del contexto en las que desarrollan sus prácticas espaciales" (Olivi, Fadda y Reyes, 2016, p. 40). Entre estas estrategias, se encuentra el acompañamiento por otras personas, a menudo mujeres (Figueroa Martinez y Forray Claps, 2015), reflejo de la interdependencia que caracteriza las prácticas de la movilidad (Jirón y Gómez, 2018).

A pesar de adoptar diferentes metodologías y enfocarse en distintas dimensiones de la movilidad, los trabajos que se enfocan en las personas mayores tienden a adoptar un enfoque cualitativo. En línea con la investigación inspirada al 'nuevo paradigma de las movilidades' (Sheller \& Urry, 2006), prevalece un enfoque en las prácticas de movilidad como objeto de investigación: como demuestra la investigación dedicada a desigualdad y movilidad urbana también en Chile, esta perspectiva permite considerar las implicancias de movilidades desiguales para la vida cotidiana de los individuos, considerando las características de una persona, sus redes relacionales y el lugar en donde uno vive (Figueroa Martínez \& Waintrub, 2015; Imilan, Jirón e Iturra, 2015; Jirón, 2010; Jirón y Gómez, 2018; Jirón \& Mansilla, 2014; Jouffe y Lazo Corvalán, 2010). Al mismo tiempo, es posible destacar la falta de enfoques cuantitativos, que por ejemplo describan la estructura socio-espacial de una ciudad, la distribución de sus habitantes, sus flujos de movilidad y patrones de accesibilidad: estos enfoques se encuentran en trabajos dedicados al desajuste espacial entre residencias y trabajos que afecta todos los grupos socioeconómicos y especialmente los grupos de bajos ingresos (Cox y Hurtubia, 2016; Figueroa Martínez, Hodgson, Mullen, \& Timms, 2018; Iglesias, Giraldez, Tiznado-Aitken, \& Muñoz, 2019; Niehaus, Galilea \& Hurtubia 2016; TiznadoAitken, Muñoz \& Hurtubia, 2018). Además, en el caso de la movilidad de las personas mayores prevalecen trabajos dedicados a la ciudad de Santiago y a diferentes contextos barriales, mientras que la investigación dedicada a temas de movilidad y equidad sí tiende a considerar distintos contextos urbanos del país (Vecchio, Tiznado-Aitken \& Hurtubia, 2020).

El uso de métodos cualitativos refleja el creciente interés de las ciencias sociales por las formas cotidianas de movilidad y accesibilidad de las personas mayores, considerando la edad como un relevante predictor de vulnerabilidad (Nordbakke, 2013; Nordbakke \& Schwanen, 2014; Meijering, Hoven \& Yousefzadeh, 2019; Ryan, Wreststrand \& Schmidt, 2019). Sin embargo, los enfoques cualitativos y de escala micro de las ciencias sociales todavía tienen interacciones limitadas con los enfoques cuantitativos y agregados de la ingeniería de transporte, tradicionalmente a cargo de planes y políticas para la movilidad urbana. La planificación del transporte recién ha empezado a considerar de qué manera los sistemas de transporte impactan el bienestar de los individuos, considerando en cuál medida la movilidad contribuye al acceso a oportunidades urbanas relevantes y permite contrastar distintas formas de exclusión social (Lucas, 2012). Este interés creciente ha llevado a una renovada 
atención para los análisis de accesibilidad, con un enfoque en las actividades consideradas necesarias para no ser socialmente excluidos (Lucas, Wee \& Maat, 2016), una atención específica para los grupos socioeconómicos, demográficos y étnicos en riesgo de exclusión, y un énfasis en los sistemas de transporte público (Bocarejo \& Oviedo, 2012). Debido a su naturaleza agregada, las evaluaciones de accesibilidad no logran considerar de manera eficaz características, necesidades y preferencias de distintos grupos (una excepción es Steiniger, Fuentes, Villegas, Ardiles, Rojas y Poorazizi, 2017), mientras que los tradicionales instrumentos de la planificación de transporte (por ejemplo, las Encuestas Origen-Destino y Análisis Costos-Beneficios) reiteran un enfoque en la planificación de la movilidad basada en la demanda más que en la necesidad de quienes habitan un territorio (Martens, 2006). A pesar de varios llamados para investigar y planificar la movilidad con distintas metodologías (Büscher, Sheller \& Tyfield, 2016; Pucci \& Vecchio, 2019; Sheller \& Urry, 2006), todavía es difícil superar las barreras disciplinarias y la misma planificación del transporte sigue basándose en el conocimiento cuantitativo originado por áreas de ingeniería y economía (Schwanen, 2018).

Considerando la necesidad de entender la movilidad de las personas mayores y tomarla en cuenta a la hora de tomar decisiones de planificación, los enfoques que prevalecen para el estudio del fenómeno tienen un potencial no del todo aprovechado. Las aproximaciones cualitativas mencionadas anteriormente permiten entender la movilidad a una escala de proximidad, observando las diferentes variables -individuales y de contexto- que determinan la posibilidad de moverse y analizando el impacto de la movilidad en el bienestar de las personas mayores. Sin embargo, los enfoques que consideran las implicancias sociales de la movilidad -analizando cómo diferentes y desiguales formas de movilidad aportan a la posibilidad de acceder oportunidades relevantes y, por ende, participar en la vida de una sociedad- todavía tienen un limitado impacto sobre planes y políticas para el transporte. Los tomadores de decisiones públicos usualmente no intervienen sobre el transporte como herramienta para reducir la exclusión social (Lucas, 2012), debido a la gran cantidad de datos necesarios, a las dificultades de conocer realmente las distintas exigencias de movilidad de diferentes grupos y a los costos que implican estos enfoques (Lucas, Mattioli, Verlinghieri \& Guzman, 2016; Vecchio, 2020). Estas limitaciones aplican también a Chile y a las personas mayores específicamente, que han sido beneficiadas por pocas acciones relacionadas con movilidad y acceso a oportunidades: entre ellas, la introducción de pases a tarifa reducida para el transporte público y la provisión de servicios de cuidado a domicilio por parte de algunas municipalidades. Considerando las limitaciones que políticas públicas y herramientas de análisis tienen para considerar la movilidad de las personas mayores, la adopción de metodologías mixtas dedicadas sería necesaria para desarrollar planes y políticas urbanas que desde ahora puedan considerar las exigencias de una población cada vez más anciana.

\section{Metodología}

El trabajo enfoca su análisis en el barrio San Eugenio, localizado en la comuna de Santiago (que corresponde al centro del área metropolitana de Santiago de Chile, con más de 6 millones de habitantes). El barrio se encuentra en el margen suroccidental de la comuna, en el límite con las comunas de Pedro Aguirre Cerda y Estación Central (Figura 1). El barrio, nacido a finales del siglo XIX alrededor de una maestranza de la Empresa Ferrocarriles del Estado, tuvo su auge gracias a diferentes empresas activas en su alrededor. Sin embargo, el barrio San Eugenio hoy en día tiene un sentido distinto, en el cual destacan su carácter patrimonial y residencial (Figura 2). Entre los 10.485 habitantes hay una presencia relevante de personas mayores, con el 15,9\% de la población que tiene 60 años o más (CEDEUS, 2019).

El trabajo presentado en este artículo posee un enfoque mixto que se ha desarrollado como parte de un proceso de planificación participativa para el desarrollo del Plan Integral de Movilidad de la Comuna de Santiago, en el cual han participado vecinos y vecinas del barrio San Eugenio, la Unidad de Movilidad de la Municipalidad de Santiago y el Centro de Desarrollo Urbano Sustentable (CEDEUS), centro de investigación incorporado en la 
Figura 1

Concentración espacial de adultos mayores en el área metropolitana de Santiago de Chile y ubicación del barrio San Eugenio.

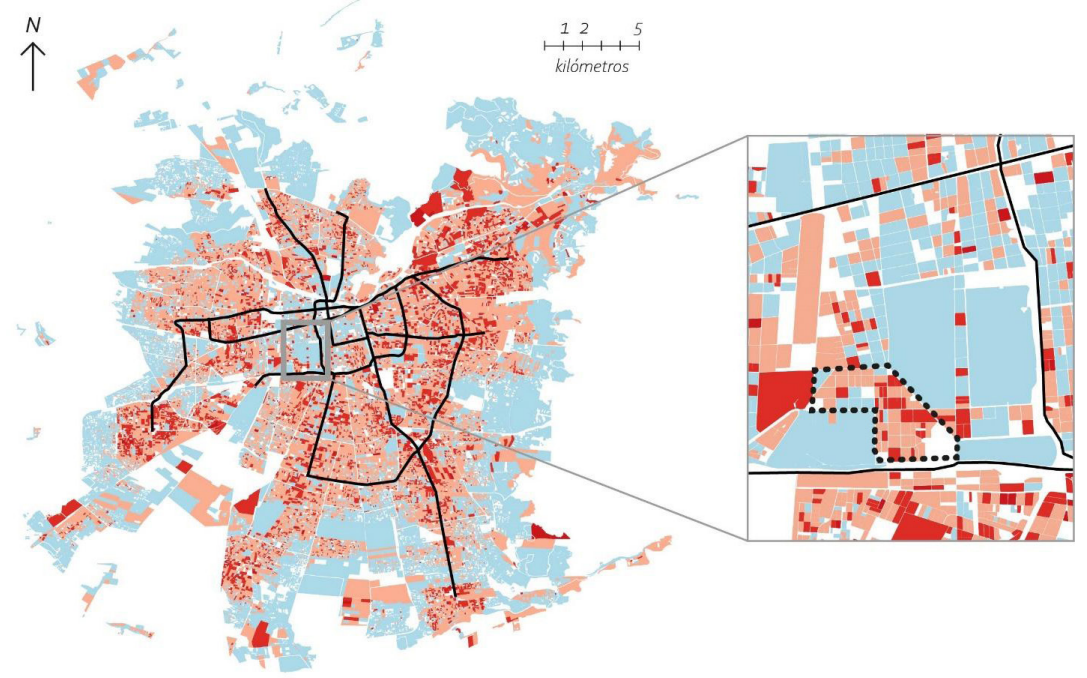

Concentración espacial de adultos mayores

$$
\begin{aligned}
& <10 \% \quad-\text { Red de metro existente } \\
& 10 \%-20 \% \\
& 20 \%-30 \% \\
& >30 \%
\end{aligned}
$$

Fuente: Elaboración propia basada en Instituto Nacional de Estadísticas, 2017.

Figura 2

Imágenes del barrio San Eugenio
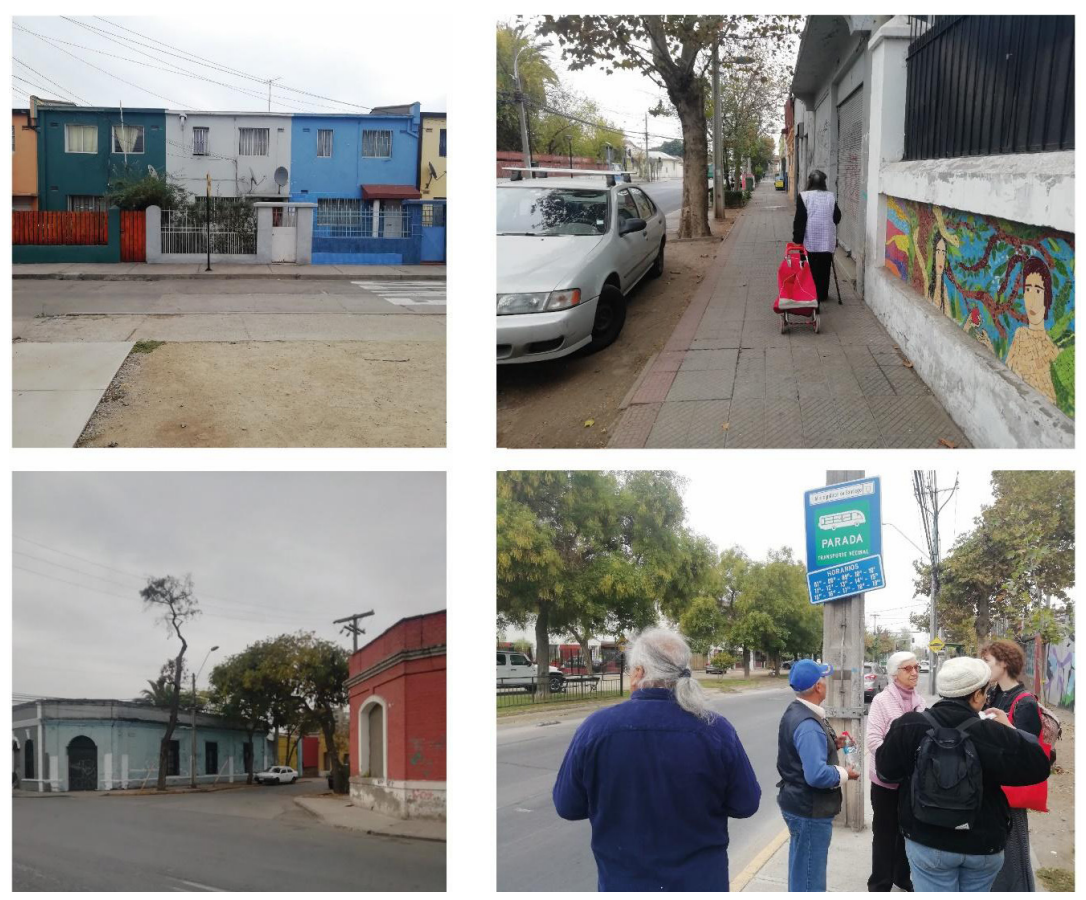

Fuente: Elaboración propia. 
Pontificia Universidad Católica de Chile y en la Universidad de Concepción.

La caracterización de movilidad y acceso de personas mayores en el barrio se ha basado en tres etapas. La primera consiste en un análisis secundario de la EOD 2012 para Santiago (Universidad Alberto Hurtado, Observatorio Social, 2014). La segunda etapa se basa en la aplicación de una encuesta de movilidad por hogar, específicamente elaborada para el barrio San Eugenio, aplicada en los meses de enero y febrero de 2019. El instrumento (véase CEDEUS, 2019) fue aplicado encuestando 354 personas, mayores de 18 años y miembros permanentes de los hogares. El objetivo de la encuesta fue caracterizar la movilidad de los habitantes del barrio, considerando sus desplazamientos dentro y fuera del mismo, así como sus elecciones modales. Además, este instrumento permitió recolectar elementos sobre la percepción que los habitantes tienen del barrio, de sus espacios públicos y de las oportunidades urbanas disponibles a nivel local. La tercera etapa se basa en una serie de entrevistas semiestructuradas a 23 personas mayores, también residentes en el barrio, con preguntas finalizadas a identificar necesidades, oportunidades, barreras y estrategias de movilidad y acceso de personas mayores. Los entrevistados, 15 mujeres y 8 hombres tienen entre 61 y 90 años, son jubilados y 7 de ellos (6 mujeres y un hombre) reportan alguna discapacidad que reduce su movilidad; todos han vivido por mucho tiempo en el barrio San Eugenio y 16 entrevistados declaran haber residido en el barrio por más de 40 años. Específicamente, la encuesta fue realizada para explorar destinos frecuentemente visitados y estrategias de movilidad (elección modal y elección de rutas), con un enfoque en los desplazamientos necesarios para cumplir con necesidades relacionadas con trabajo, salud, comercio, realización de trámites y recreación.

\section{Movilidad y acceso en el barrio San Eugenio: tres narraciones}

A continuación, se describen brevemente los resultados que emergen desde las tres fuentes descritas anteriormente.
La movilidad de los habitantes del barrio en la EOD 2012. La EOD 2012 para Santiago (Universidad Alberto Hurtado, Observatorio Social, 2014) ofrece una primera aproximación de manera agregada a la movilidad en el barrio San Eugenio. Las EOD son elaboradas por el Ministerio de Transportes y Telecomunicaciones de Chile con el fin de planificar y gestionar proyectos de inversión en infraestructura de transporte al nivel de ciudad. Esta encuesta se repite cada 10 años y está disponible solo para las ciudades de grande o medio tamaño. Nuestro análisis de la EOD del área metropolitana de Santiago considera todos los viajes, no solo de adultos mayores, debido a un número inferior de adulto mayores encuestados: solo 5 de 84 entrevistas realizadas (Tabla 1). En general, las zonas de la encuesta que incluyen el barrio originan prevalentemente viajes por trabajo o estudio. Considerando la muestra analizada, los modos prevalentes son la caminata (35\%), el transporte público, incluyendo buses troncales y alimentadores, el Metro y taxi colectivo (aprox. $35 \%$ ), y el auto (20\%). La gran mayoría de los viajes desde el barrio tiene su destino en la misma comuna de Santiago, mientras que los viajes intercomunales se dirigen sobre todo a las comunas de Estación Central y Maipú, y en mesura menor a Pedro Aguirre Cerda, Providencia y Puente Alto (Figura 3).

Tabla 1

Estadísticas de la Encuesta Origen-Destino 2012 para el barrio San Eugenio.

\begin{tabular}{lll} 
& Entrevistados & Personas mayores \\
\hline Personas encuestadas & 84 & 5 \\
\hline Viajes totales & 106 & 5 \\
\hline Viajes intercomunales & 43 & 0 \\
\hline Viajes intracomunales & 63 & 5 \\
\hline
\end{tabular}

Fuente: Elaboración propia.

El nivel de agregación de la Encuesta Origen-Destino no permite una caracterización de la movilidad en el barrio, impidiendo también un enfoque en las personas mayores. Si bien la finalidad de la EOD es una caracterización de la movilidad a nivel de ciudad a largo plazo (con un horizonte temporal de 10 años), la mirada que esta ofrece termina siendo limitada a los destinos alcanzados por las personas que se desplazan, el propósito de sus viajes y el modo de 


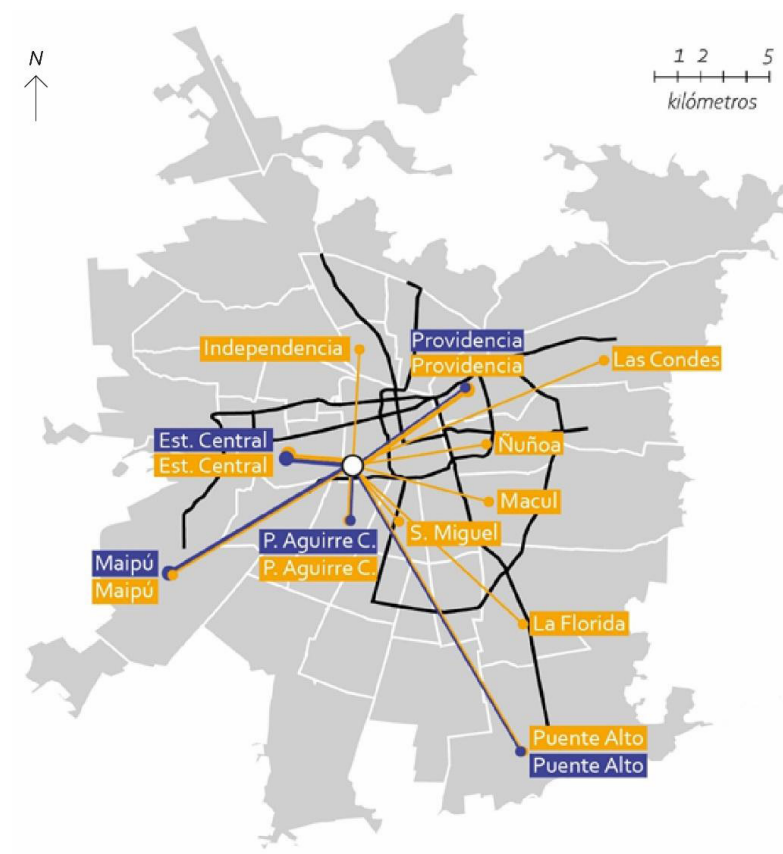

Principales destinos de los viajes originados desde el barrio San Eugenio

viajes en la Encuesta Origen-Destino

viajes en la Encuesta por Hogares Cedeus

Red de metro existente

Fuente: Elaboración propia.

transporte que estas ocupan. La principal finalidad es representar flujos de personas en movimiento, asumiendo que en sus desplazamientos tratan de minimizar los costos monetarios y temporales del viaje. Esto lleva a no considerar las prácticas de movilidad de cada persona, dejando de lado por ejemplo la experiencia que cada persona hace en un cierto espacio (qué ruta elige, cómo percibe los lugares, cómo se siente al moverse) y cómo las características del contexto facilitan o impiden la movilidad de cada persona. En las EOD "la ciudad, con todas sus obstinadas materialidades, desaparece; solo las poblaciones en movimiento son relevantes" (Ureta, 2015, p. 32), con un enfoque limitado que no permite entender las dinámicas de movilidad a una escala barrial y no captura las características de contexto que influyen en la movilidad de distintos grupos.

La movilidad de los habitantes del barrio en la Encuesta por hogar CEDEUS 2019. La Encuesta por Hogares realizada por el centro de investigación CEDEUS (EHC) en el 2019 (véase CEDEUS, 2019) muestra de manera más detallada la movilidad del barrio San Eugenio (tabla 2). Un primer elemento de atención es la composición de los viajes intra-comunales e intercomunales (entre comunas que componen la ciudad de Santiago. Los viajes al interior de la misma comuna prevalecen, sobre todo en el caso de viajes relacionados con trabajo y estudio (55\% de los viajes para este propósito son intra-comunales), así como con compras, tramites y salud (tres viajes de cada cuatro son intra-comunales). Sin embargo, la encuesta muestra una geografía más compleja, en comparación con la EOD 2012, con respeto a los viajes intercomunales. En la EOD prevalecían comunas confinantes y cercanas al barrio, como Estación Central y Pedro Aguirre Cerda, además de Providencia y, al sur de Santiago, Maipú y Puente Alto (Figura 3). En la EHC 2019, entre las comunas de destino para viajes relacionados con trabajo y estudio emergen Providencia, Las Condes y Ñuñoa, que forman parte del 'cono de alta renta' en el sector oriente de Santiago hacia el cual se dirige la mayoría de los viajes por trabajo de la ciudad (Garretón, 2012; Link, Valenzuela y Fuentes, 2015). Estación Central emerge como una polaridad para realizar compras y trámites, mientras que Providencia es el segundo destino para los viajes relacionados con servicios de salud. Los viajes para visitar a la familia y amigos resultan más complejos, involucrando principalmente la parte sur de la ciudad de Santiago. Los viajes para actividades no obligatorias resultan particularmente relevantes para entender la movilidad de las personas mayores, quienes representan el 40\% de los encuestados y cuya movilidad no era analizable utilizando los resultados de la EOD.

Tabla 2

Estadísticas de la Encuesta por hogares de CEDEUS para barrio San Eugenio.

\begin{tabular}{lll} 
& Entrevistados & Personas mayores \\
\hline Personas encuestadas & 354 & 144 \\
\hline Viajes total & 301 & 114 \\
\hline Viajes intercomunales & 84 & 23 \\
\hline Viajes intracomunales & 217 & 91 \\
\hline $\begin{array}{l}\text { No Responde/ No } \\
\text { Aplica }\end{array}$ & 53 & 30 \\
\hline
\end{tabular}

Fuente: Elaboración propia. 
Figura 4

Destinos y actividades de las personas mayores entrevistadas (arriba: en la ciudad de Santiago; abajo: en el barrio San Eugenio).
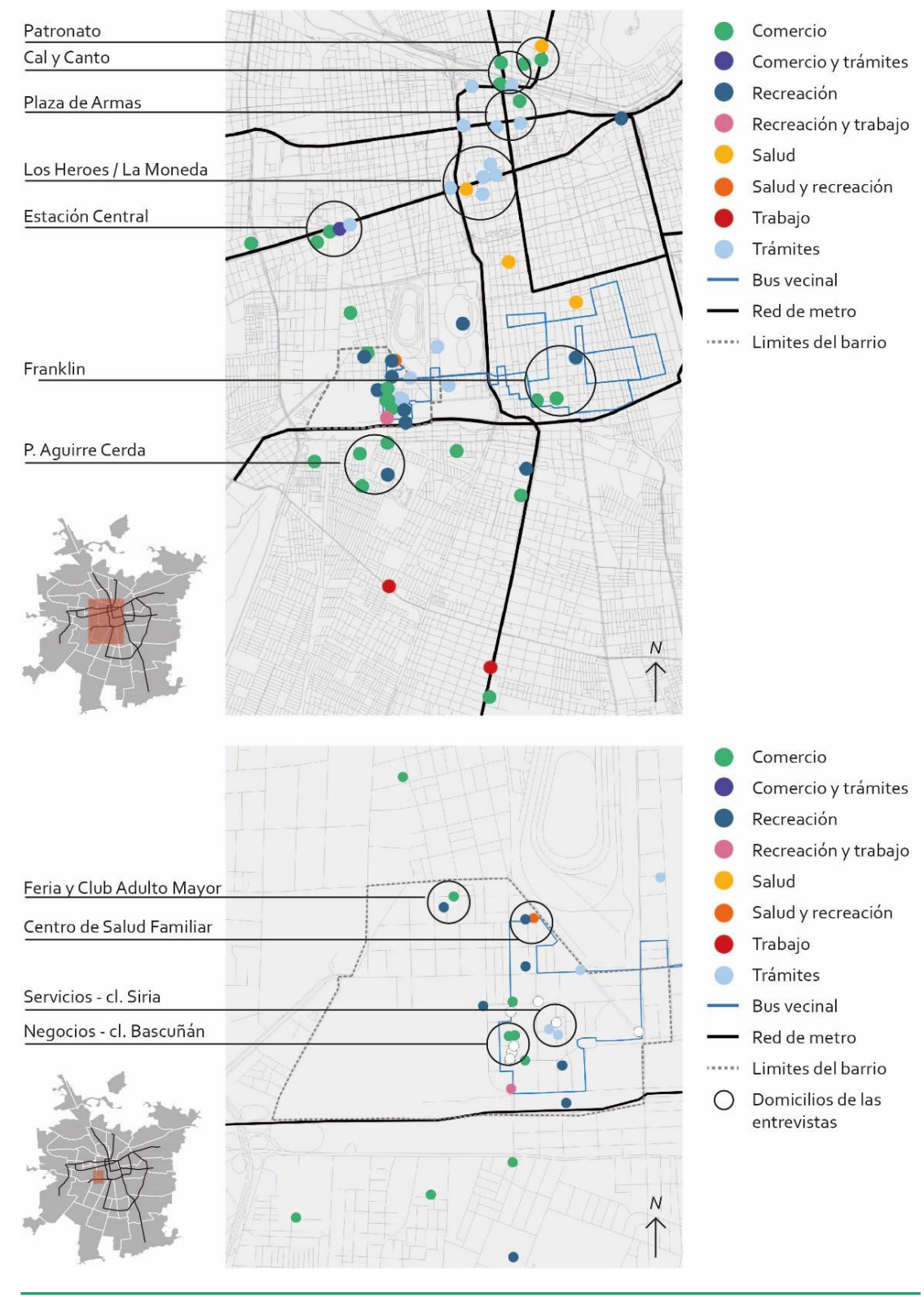

Fuente: Elaboración propia.

Entre los medios de viaje, el bus urbano emerge como la opción principal para la mayoría de los viajes, sean para actividades obligatorias (ir al trabajo o a la escuela) o no obligatorias (realizar compras, recibir atención de salud, realizar trámites y visitar familiares o amigos). El Metro resulta la segunda opción principal para viajes cotidianos al trabajo o a la escuela, mientras que la caminata resulta el segundo medio preferido en el caso de viajes relacionados con compras (29\%) y salud (32\%), que ocurren principalmente al interior de la misma comuna. Las elecciones modales son probablemente relacionadas con las oportunidades disponibles en el barrio, que incluyen, según los encuestados, sobre todo centros médicos públicos, establecimientos educacionales, farmacias, sedes sociales y centros deportivos. Finalmente, de la EHC emergen también algunas prácticas de encadenamiento de viajes: en la mayoría de los casos, viajes por distintas razones son también la ocasión para realizar compras. 
En particular, emerge una relación significante entre la realización de compras y trámites, así como los viajes por trabajo y estudio son aprovechados también para comprar y realizar trámites.

Tratándose de un análisis enfocado en el barrio de estudio, la EHC muestra algunas ventajas para entender la movilidad del barrio San Eugenio. Los resultados obtenidos permiten una caracterización más detallada de las maneras en que los habitantes de la zona se mueven, aunque esto no se debe exclusivamente al hecho de haber pensado e implementado la encuesta a nivel de barrio. Un elemento fundamental de la encuesta es la combinación de preguntas enfocadas en las prácticas de movilidad (destinos y propósitos de los viajes, modos de transporte utilizados...) y en la percepción del barrio, sobre todo en términos de oportunidades disponibles a su interior. De esta manera, es posible entender en qué medida el barrio analizado depende de otras zonas (de la comuna o de la ciudad) para la realización de actividades básicas. Adicionalmente, el diseño de las preguntas permite también destacar algunas formas de encadenamiento de viajes. El foco en un barrio y el diseño de la muestra permiten entonces una representación más detallada de la movilidad local, permitiendo también un enfoque en las personas mayores. Sin embargo, el desarrollo de una encuesta dedicada resulta dispendioso considerando los recursos necesarios para su diseño, implementación y análisis, lo que impide la adopción generalizada de encuestas barriales como herramienta para la planificación y sugiere su aplicación en contextos seleccionados pero representativos, por ejemplo, en caso de barrios que presenten fuertes problemas de movilidad y dificultad en el acceso a oportunidades.

La movilidad de los adultos mayores del barrio en las entrevistas CEDEUS 2019. Las entrevistas con adultos mayores (EAM) realizadas por CEDEUS en el 2019 (véase CEDEUS, 2019) se enfocaron en conocer las prácticas de movilidad de este grupo demográfico en el barrio. Los resultados permiten analizar tanto los destinos alcanzados como también los motivos de viaje y las elecciones modales, distinguiendo entre la escala del barrio, de la comuna y de la ciudad.
El análisis de las entrevistas revela que, a nivel de barrio prevalecen los viajes para realizar compras y trámites; se registran también viajes con el propósito de llegar a lugares de recreación y, en algunos casos, a servicios de salud. Los destinos se concentran sobre todo en las calles principales del barrio y en general en los ejes más recorridos, por ejemplo, donde hay servicios de transporte público y especialmente un bus vecinal gratuito para los vecinos del barrio.

La mayoría de los destinos se encuentran en la misma comuna de Santiago, con polos que tienen diferentes especializaciones y que son alcanzados principalmente en Metro (Figura 4). Por ejemplo, en la zona céntrica de Los Héroes - La Moneda, la cual se encuentra a 30 minutos del barrio en bus y en Metro, se realizan trámites (relacionados con jubilación) y se accede a servicios especializados de salud. En el corredor Plaza de Armas - Estación Mapocho (35 minutos en bus y Metro), parte del centro cívico de la ciudad, se concentran servicios y comercio mayor y es visitado por las personas mayores entrevistadas para satisfacer varias necesidades en un sólo viaje, como trámites de todo tipo y compras. En proximidad de esta zona se encuentran el nodo de Cal y Canto, con zonas dedicadas al comercio (por ejemplo, la Vega, el principal mercado de la ciudad), y el barrio Patronato, en donde se desempeñan también actividades relacionadas con trámites y se accede a servicios de salud. La zona alrededor de la Estación Central, que se encuentra un poco más lejos de centro cívico de Santiago, es visitada prevalentemente en micro para realizar compras de supermercado y otros trámites. Las personas mayores se mueven en bus también hacia el oriente y el sur del barrio, para alcanzar otros barrios cercanos como Franklin, Matta y Almagro, en que se satisfacen diferentes necesidades de abastecimiento (sector Franklin y Biobío) y salud (Hospital San Borja Arriarán).

En comparación con las encuestas, las entrevistas permiten no solo mapear los viajes de las personas mayores, incluyendo modo y propósito de viaje, sino también considerar sus prácticas de movilidad y sus experiencias en movimiento. En este sentido, un elemento que 
emerge con fuerza desde las entrevistas son los conflictos relacionados con la experiencia del movimiento, tanto en el espacio público como también en los específicos espacios de la movilidad.

Con respecto a los conflictos en el espacio público los entrevistados se refieren principalmente a la condición de espacios destinados al movimiento como por ejemplo las veredas. Varios entrevistados señalan el mal estado de las veredas, reportando las consecuencias que han tenido para personas con limitada autonomía de movimiento o en no perfectas condiciones de salud:

Hay veredas que no están muy buenas. Pero no tanto. Uno cuando tiene que pasar se las arregla o busca el lado. $\mathrm{Y}$ ahí en mi calle igual, hay hoyos y las veredas levantadas, entonces es problema para la gente de la tercera edad. Si ahí se han caído personas con la silla de ruedas y se han dado vuelta. Hay varios datos así. (mujer, 72 años)

Andaban por ahí viendo que según como me decía mi marido que parece que van a emparejar las veredas. Mi marido se ha caído cuántas veces. Yo le digo “Estai viejo, ya ni te afirmai siquiera”. (mujer, 79 años)

Según los entrevistados la mala condición de las veredas se puede atribuir tanto a falta de mantención como también a presencia de camiones de carga que estacionan sobre las veredas (opinión confirmada por visitas en terreno de los autores; véase Figura 5).

Un segundo elemento son los conflictos relacionados con el transporte y la experiencia de desplazarse ocupando el transporte público. Por un lado, hay elementos problemáticos relacionados con el diseño de la red de transporte público, que después de la introducción del sistema de transporte público integrado Transantiago ha privilegiado algunas relaciones (por ejemplo, conexiones radiales) constriñendo a adaptarse a una red menos flexible y consecuentemente a recorridos más complicados (Jirón, Imilan e Iturra, 2016). Por ejemplo, un entrevistado comenta sobre los servicios de transporte público:
Figura 5

El estado de las veredas hace que en algunos casos resulte más fácil caminar por la calle.

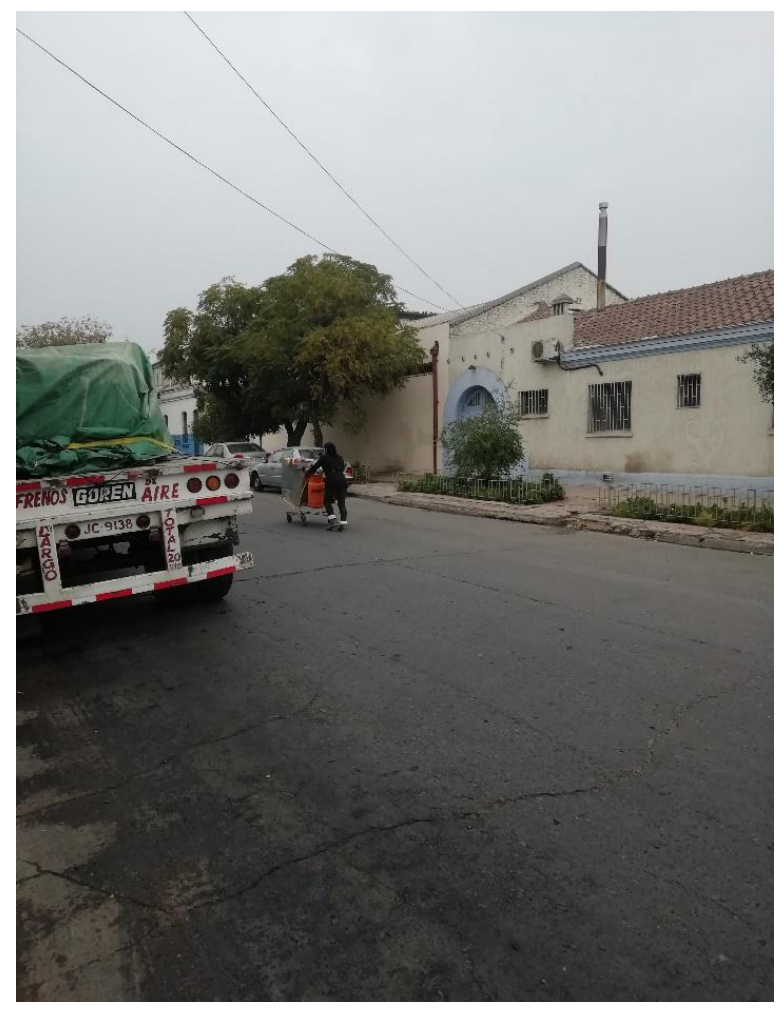

Fuente: Elaboración propia.

Que te dejan lejos no más, lejos de donde vai tu. Por ejemplo, yo tengo que caminar de [la intersección] Bandera con General Mackenna hasta el municipio [de Santiago]. Si te vai en Metro también es complicado porque van todos llenos y hay que hacer 2 combinaciones. (hombre, 64 años)

Por otro lado, la misma experiencia del viaje puede ser percibida como insegura y no agradable por adultos mayores, como en este ejemplo referido a los viajes en bus:

No es que a veces voy de aquí a la estación, vuelvo que anda mucha gente y la gente imprudente. A eso le tengo respeto. La gente es muy atrevida, cuando anda con bolsos grandes. (mujer, 72 años)

Un tercer elemento son los conflictos con otros modos de transporte, visibles cuando peatones, ciclistas y automovilistas comparten el mismo espacio de la calle. El principal elemento percibido como peligroso son los automóviles (Figura 6): 
Figura 6

Cruce de calles en el barrio. Es evidente el gran espacio a disposición de los automóviles, lo que facilita que éstas circulen a elevada velocidad.

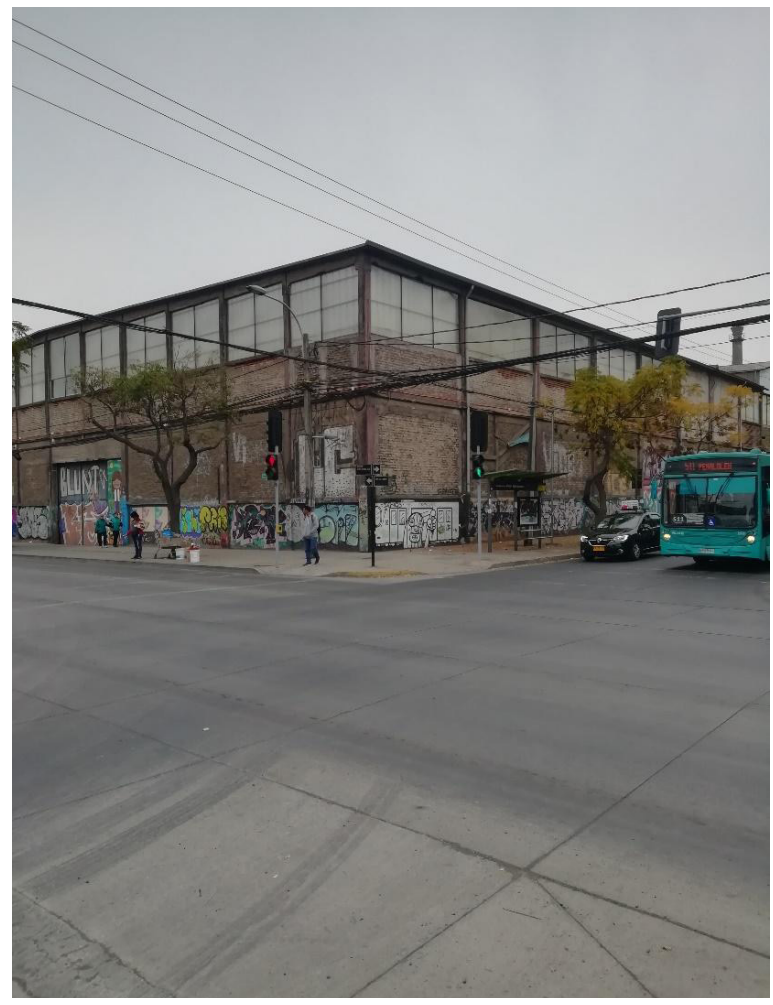

Fuente: Elaboración propia.

Si, porque si no como te digo... hay calles como Pizarro que son 4 cuadras y hay dos lomos de toro [reductores de velocidad] aquí e igual la pican, 60 $\mathrm{km} / \mathrm{h}$ aquí. Bascuñán [la calle] $80 \mathrm{o} 100 \mathrm{~km} / \mathrm{h}$ porque es más recta. Lo que pasa es que todos los flujos de las poblaciones son aquí y después parten al otro lado, por Bascuñán que es al otro lado, entonces (...) Claro, que se señalizara en esta parte de aquí que son $1,2,3,4,5,6,7,8,9,10$ cuadras que no corran a gran velocidad... Como te digo, porque no funcionan los lomos de toro. Entonces habría que regular en estos ejes. Por ejemplo, Pizarro... En el sentido, es que, sino en todas esas partes tendríamos que colocar semáforos, la única forma de parar. (hombre, 64 años)

La convivencia con otros modos resulta complicada también en las veredas, por ejemplo, en el caso de las bicicletas:

Es complicado porque están usando las veredas y no respetan la gente. Eso está pasando. Yo los reto y [...]son atrevidos. Oye esto no es ciclovía le digo.
Me miró y atravesó la calle en la bicicleta y chao. Así atrevido. Eso está complicado. (mujer, 72 años)

Combinando métodos para identificar estrategias de movilidad y acceso en personas mayores. La combinación de miradas cuantitativas (EOD y EHC) y cualitativas (entrevistas), con un enfoque en un barrio especifico, permite una aproximación a las estrategias de movilidad y acceso de las personas mayores. La población anciana tiende a poderse mover menos de manera autónoma, debido a distintos factores económicos y de salud, entre otros. Además, las personas mayores son a menudo involucradas en actividades de cuidado familiar (Gutierrez \& Ochoa, 2016). Sin embargo, los elementos que reducen las posibilidades de movimiento de la persona mayor no necesariamente impiden su capacidad de acceder a bienes y servicios necesarios. En el caso del barrio San Eugenio, dos elementos compensan, por lo menos parcialmente, estas limitaciones. Por un lado, la posición pericentral del barrio garantiza una buena presencia de servicios de transporte público (tanto líneas de Metro como buses) y con eso una cercanía al centro de Santiago con sus múltiples oportunidades. Por el otro, encuestas y entrevistas muestran que las personas mayores hacen uso por lo menos de tres estrategias de movilidad, descritas a continuación.

Privilegiar destinos locales. Las personas mayores del barrio San Eugenio privilegian en gran mayoría destinos localizados en el mismo barrio y en la comuna de Santiago en general. Como muestra la Figura 4, a nivel barrial emergen algunos focos de actividad, vinculados con la presencia de negocios y servicios, así como con lugares utilizados con regularidad (por ejemplo, un club de adultos mayores, una feria y un centro de salud). Las entrevistas describen con mayor detalle las prácticas de movilidad, distinguiendo entre actividades diarias desempeñadas en el mismo barrio (sobre todo alrededor de algunos polos locales) y otras que requieren desplazamientos al interior de la misma comuna, hacia lugares bien conectados (sobre todo gracias al bus vecinal y al Metro) y que ofrecen variedad de servicios. En otros contextos de la misma ciudad de Santiago, este fenómeno de 'repliegue barrial' (Jouffe, 


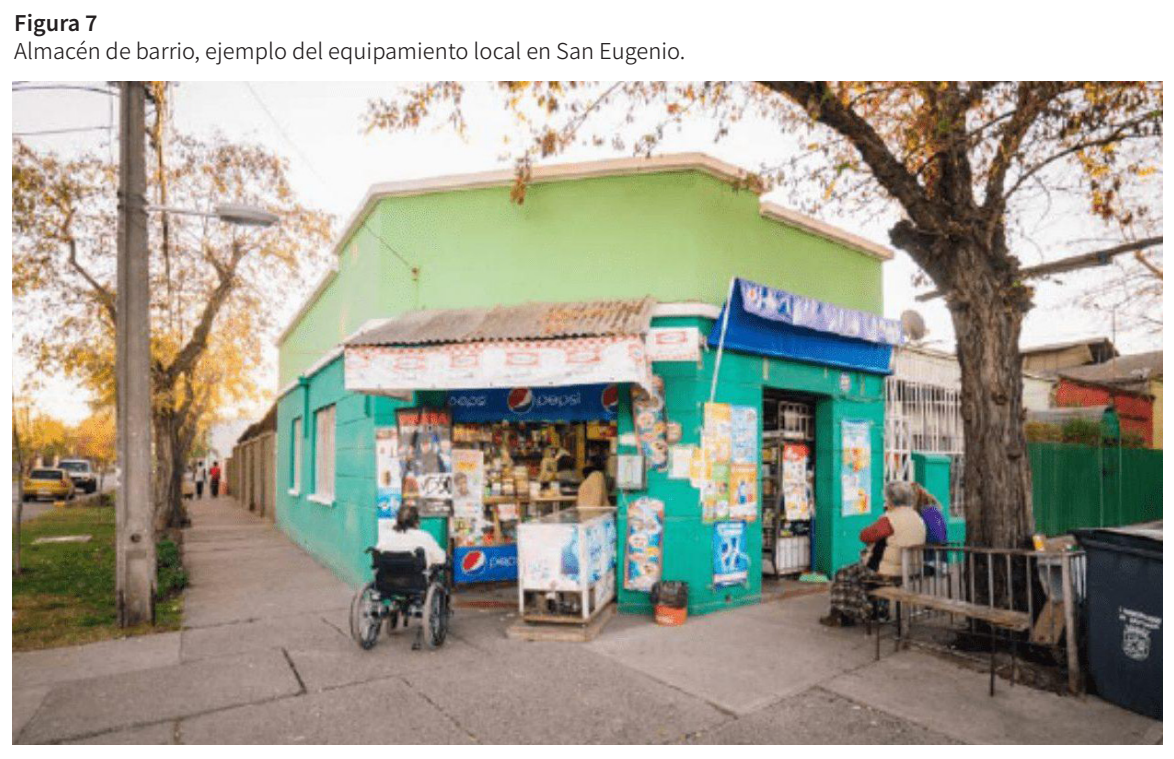

Fuente: CEDEUS, 2019.

2011) ha sido interpretado como una manera en que grupos sin muchos recursos físicos o económicos para desplazarse garantizan su acceso a oportunidades urbanas básicas, limitando su movilidad a un entorno próximo en donde es posible moverse con suficiente autonomía:

En esta calle hay almacén, carnicería, verdulería... es bien comercial este barrio. Antes era mejor todavía cuando estaba Yarur [fábrica textil], ahí sí que estaba lleno de almacenes, pero como todo eso ya terminó, es difícil tener un negocio que no sea almacén que levante: verdulería... esas cosas sí. Sino hay que ir a la Alameda a comprar ropa, pero es lo único para lo que tiene que salir uno para allá. Siempre va a la estación porque es lo más cerca. Sale mucho más caro ir al centro. (mujer, 92 años)

Sin embargo, en el caso de San Eugenio la preferencia por actividades locales es probablemente debida tanto a las favorables oportunidades de equipamiento en el barrio (Figura 7), como a la posición del barrio en la ciudad de Santiago, que permite tener al alcance lugares atractivos y actividades relevantes para el adulto mayor. En este sentido emerge una diferencia entre los resultados de las dos encuestas EOD y EHC, en las cuales los viajes intercomunales tienen una mayor incidencia, aunque en muchos casos estos están relacionados con actividades de trabajo y estudio que no se desempeñan en la tercera edad.

Combinar modos de transporte. Los relatos de las personas mayores muestran formas de intermodalidad específicas de la tercera edad. Por ejemplo, la mayoría de los destinos localizados afuera del barrio son alcanzados en Metro, a pesar de que dos líneas de buses pasen cerca del barrio. Los adultos mayores llegan al Metro utilizando un servicio de bus vecinal, gratuito para las personas de la tercera edad (Figura 8). Como mencionan algunos entrevistados,

Trato de economizar a través de la [micro] vecinal. $\mathrm{Y}$ eso porque el transporte en marzo en adelante gastó mucho por los trámites que hay que hacer en la muni asá que de ahí empiezo a ahorrar. Podrían ser sus $\$ 5.000$ a la semana. (mujer, 92 años)

Si para llegar al centro es donde complica. No hay ninguna micro. Hay que bajarse en la Alameda y hay que hacer transbordo, bajarse y hacer lo que tiene que hacer y subirse ahí mismo. Se sube al Metro y se viene a patita pa' acá. Gasta 700 pesos... es más seguro. (hombre, 72 años)

En las personas mayores el uso del Metro tiene una incidencia mayor que en los otros grupos encuestados, ya que prefieren el Metro a los autobuses. Esta preferencia por el Metro se puede explicar por un lado considerando que este medio de transporte es percibido como más rápido y seguro en comparación con los servicios de autobuses. Por otro lado, el Metro resulta económicamente más accesible que otros medios, elemento importante para personas mayores que mayoritariamente reciben bajas pensiones (Dirección de Estudios Previsionales, 2019). 


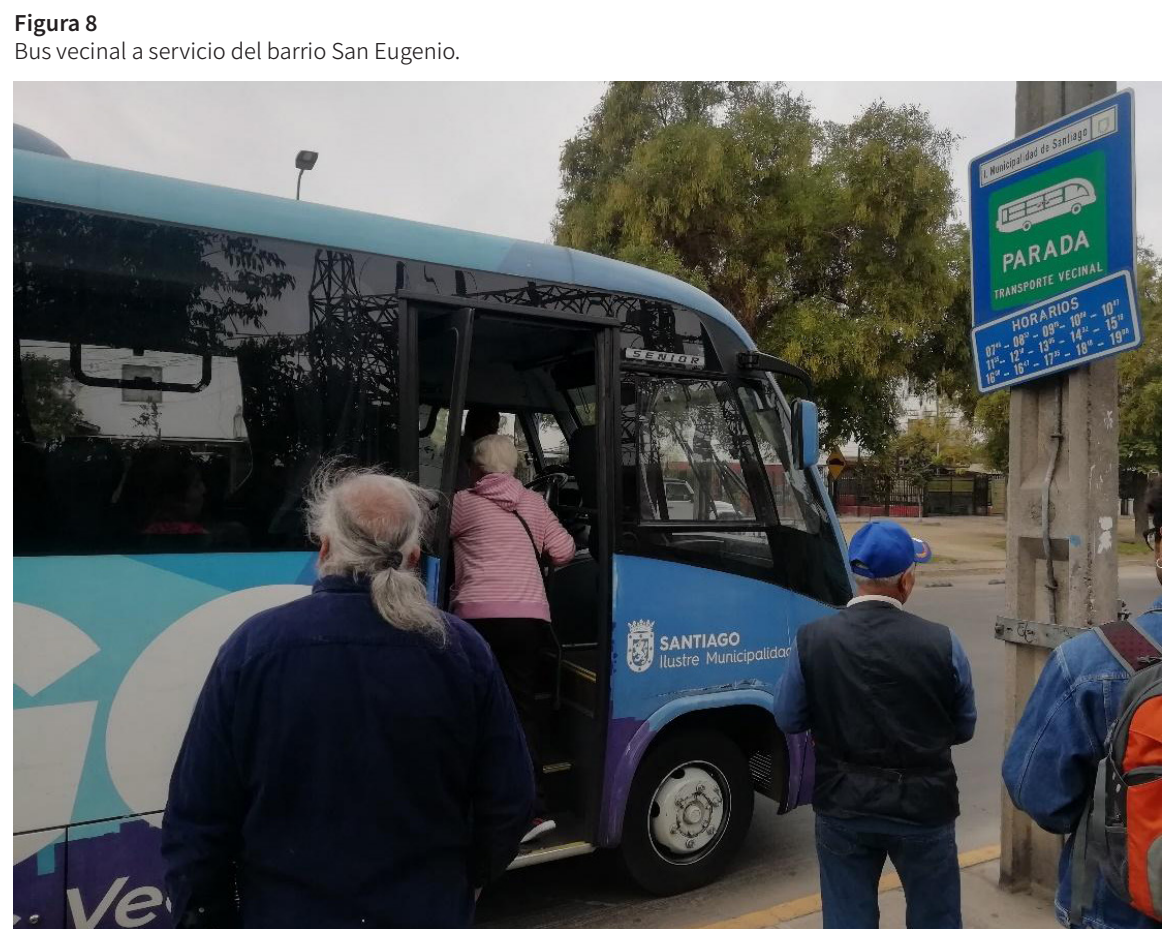

Fuente: Elaboración propia.

Gracias a la Tarjeta del Adulto Mayor (TAM), para las personas mayores es posible viajar en Metro en cualquier horario a una tarifa rebajada. Sin embargo, la TAM no aplica a otros servicios de transporte público (Vecchio, Castillo, Steiniger y Muñoz, 2019). La estrategia que combina bus vecinal y Metro muestra como las personas mayores adaptan su movilidad a los servicios existentes, escogiendo rutas menos directas, pero más económicas para lograr moverse a pesar de algunas limitaciones. Además, esta estrategia ofrece elementos para analizar los servicios de transporte publico existentes, permitiendo evaluar los beneficios de un servicio de bus vecinal y los beneficios tarifarios existentes para personas mayores.

Delegar actividades. Una tercera estrategia permite que las personas mayores accedan a bienes y servicios sin desplazarse. Un primer ejemplo se refiere al utilizo de tecnologías de información y comunicación, como el pago de cuentas por internet, aunque la baja alfabetización digital de los adultos mayores reduzca la relevancia de esta opción. Un segundo ejemplo más relevante se refiere al delegar a hijos, familiares o vecinos algunos de estos viajes. Se trata de un ejemplo de interdependencia, que demuestra como la movilidad no es una práctica solo individual, sino una red que articula varias personas que están relacionadas entre sí (Jirón y Gómez, 2018): es el caso de la abuela que cuida la nieta en casa, para que la hija pueda salir a trabajar; el caso del hijo que utiliza un servicio de ridesharing para que el padre anciano pueda salir de la casa; el caso del vecino que se encarga, gratuitamente o no, de las compras para un adulto mayor; el caso de las mismas personas mayores que se organizan para moverse juntos, por ejemplo para acompañar a personas con movilidad reducida que no se sienten seguras caminando autónomamente. Es un tema que emerge en varias entrevistas:

Yo le di el poder a mi hija de todas las cosas. Todo tipo de trámites los hace ella. Yo no salgo para nada, si parezco guagua sietemesina. Paso en la casa o aquí en la banca sentada con mi vecina. La única salida mía es a la banca. (Mujer, 69 años)

Desde que ya empecé a tener problemas para salir, no salgo, a no ser que mi hijo me lleve. (...) si yo estando acompañada no tengo problema. Tengo que ir lento eso sí, pero teniendo alguien al lado a mí me da más seguridad, más confianza que me pueda tomar cualquier cosa. (Mujer, 63 años)

[hablando de una nieta] Algunas veces la voy a buscar a su casa, a dejar a su casa. Voy a sus reuniones de colegio. Nos citan mucho porque ella está en un programa que se llama PIE. Ellos [los padres] están 
muy contentos porque nosotros le damos apoyo en la casa. Porque podemos afortunadamente. (Mujer, 69 años)

Delegar desplazamientos y actividades es entonces una manera de limitar los efectos negativos generados por la reducida movilidad, permitiendo superar barreras que un individuo, solo, no podría enfrentar (Demoraes, Contreras y Piron, 2016).

Las tres estrategias muestran la importancia de combinar métodos para entender la movilidad de las personas mayores con un nivel de detalle no posible con otras herramientas. Considerando que la movilidad de las personas mayores se caracteriza por la prevalencia de destinos locales y de actividades consideradas como "no obligatorias" (es decir, no vinculadas a trabajo y estudio), el desarrollo de instrumentos de investigación dedicados es relevante para complementar los resultados -a menudo parciales- de otras herramientas agregadas de análisis. Además, se observa como el uso de entrevistas permite una representación más precisa de las prácticas de movilidad de las personas mayores. Por un lado, se detectan estrategias adaptivas, especialmente relevantes para mostrar cómo la persona mayor enfrenta los posibles obstáculos a su autonomía de movimiento, sean barreras económicas, espaciales o de salud (Olivi et al., 2016). Por el otro, el carácter semiestructurado de la entrevista da el espacio para que el entrevistado no solo reporte una estrategia de movilidad, sino también explique los factores que la motivan, ofreciendo indicaciones más exactas para la acción pública. Por ejemplo, un servicio de buses vecinales puede ser preferido porque es gratuito o por su comodidad, señalando así la existencia de barreras económicas o físicas. De la misma forma, el uso de herramientas cualitativas permite reconstruir las formas de interdependencia que, a pesar de su relevancia, resultan difíciles de reconstruir utilizando exclusivamente herramientas tradicionales de la planificación de transporte (Jirón et al., 2020). Finalmente, un enfoque barrial permite también un diseño de la muestra de entrevistados que permita incluir personas con movilidad reducida, para los cuales la interdependencia resulta fundamental a la hora de enfrentar necesidades cotidianas sin poder o tener que moverse de su casa.

\section{Conclusiones}

El envejecimiento de la población y su impacto sobre las ciudades tiene relevantes implicancias para el abordaje de los problemas urbanos y de la movilidad. Desde su enfoque barrial, el trabajo presentado es un primer acercamiento al tema, mostrando que combinar diferentes métodos de investigación puede ser relevante para trabajar en base a los problemas de la movilidad de las personas mayores. Reconstruyendo la contribución de distintas herramientas de análisis y mostrando la complementariedad de sus resultados, se evidencia la relevancia de un enfoque interdisciplinario, que sin embargo no han sido objeto de una atención específica a la hora de planificar la movilidad urbana.

En primer lugar, se observan las limitaciones de los instrumentos tradicionales de análisis a la hora de considerar la movilidad y el acceso para las personas de la tercera edad. Las herramientas en que normalmente se basa la planificación urbana del transporte, como las Encuestas Origen-Destino, resultan limitadas a la hora de considerar la accesibilidad disponible para los adultos mayores: (1) representan los viajes realizados pero no ofrecen elementos sobre las experiencias en movimiento (tanto en el espacio público como en los medios de transporte), ni sobre las estrategias desempeñadas para adaptarse a los vínculos de los sistemas existentes (por ejemplo, beneficios tarifarios limitados solo al Metro en el caso de Santiago, por lo menos hasta el año 2020; véase Vecchio et al., 2019); (2) no muestran los viajes que la persona mayor no realiza por razones que salen de la encuesta; (3) no representa la interdependencia entre la persona mayor y sus redes relacionales familiares y vecinales. La prospectiva interdisciplinaria empleada en este trabajo plantea la necesidad de combinar métodos cuantitativos y cualitativos para una mejor aproximación a las prácticas de movilidad, como es demostrado también en el contexto chileno - en relación con las barreras de movilidad asociadas por ejemplo a género e interdependencia (Jirón et al., 2020). 
El enfoque en las personas mayores muestra la necesidad de ocupar diferentes herramientas de análisis. Herramientas cuantitativas permiten representar el contexto más general en que se coloca la movilidad de la persona mayor, comparándola con las dinámicas de movilidad de otros grupos de la misma población. Por otro lado, enfoques cualitativos son necesarios para capturar informaciones más específicas que permiten representar las diferentes prácticas de movilidad y acceso, y para conocer con más detalle el contexto local en el cual se va a planificar. Si bien la complejidad y la multiplicidad de las movilidades contemporáneas requieren enfoques multidisciplinarios (Pucci \& Vecchio, 2019b), resulta complejo estructurarlos de manera tal que sea posible replicarlos en contextos diferentes. Adicionalmente, estos enfoques tienen un elevado costo económico de implementación, lo que limita el tipo de actores institucionales y no - que puedan efectivamente utilizar estas herramientas. Debido a los costos, es posible pensar también a una aplicación de estos instrumentos que se concentre en específicos grupos o contextos. En una perspectiva de 'transporte justo' (Martens, 2016), sería necesario enfocarse en territorios que padezcan de escasos niveles de accesibilidad, para entender con mayor detalle las necesidades de sus habitantes y las formas de movilidad a su disposición.

El punto anterior evidencia la importancia de la replicabilidad (y escalamiento) de este enfoque interdisciplinario. A pesar de un creciente interés para el envejecimiento de la población y su dimensión territorial en el contexto latinoamericano (Sánchez-González \& Rodríguez-Rodríguez, 2016), no se han desarrollado programas multidimensionales capaces de enfrentar las necesidades de movilidad y acceso de las personas mayores o de adaptar las ciudades para una población que a futuro será cada vez más anciana. Mientras la investigación y la práctica profesional muestran cada vez más atención para las implicancias sociales de la movilidad y como considerarlas en la planificación del transporte (Pucci \& Vecchio, 2019a), es necesario que las instituciones responsables de la gestión de las ciudades reconozcan explícitamente que el (re)diseño de los espacios públicos y de los sistemas de movilidad es clave para el bienestar de la población anciana. En este sentido, la información levantada con el enfoque interdisciplinario propuesto tiene que ser el primer paso hacia la definición de acciones que puedan garantizar la movilidad, y consecuentemente el bienestar, de las personas mayores.

\section{Referencias bibliográficas}

Akhavan, M., \& Vecchio, G. (2018). Mobility and Accessibility of the Ageing Society. Defining Profiles of the Elderly Population and Neighbourhood. TeMA - Journal of Land Use, Mobility and Environment, 9-22. https://doi. org/10.6092/1970-9870/5757

Banister, D., \& Bowling, A. (2004). Quality of life for the elderly: The transport dimension. Transport Policy, 11(2), 105-115. https://doi.org/10.1016/S0967-070X(03)00052-0

Bilal, U., Alazraqui, M., Caiaffa, W. T., Lopez-Olmedo, N., Martinez-Folgar, K., Miranda, J.J., Rodriguez, D.A., Vives, A., \& Diez-Roux, A. V. (2019). Inequalities in life expectancy in six large Latin American cities from the SALURBAL study: an ecological analysis. The Lancet Planetary Health, 3(12), 503-510. https://doi.org/10.1016/ S2542-5196(19)30235-9

Bocarejo, J. P., \& Oviedo, D. (2012). Transport accessibility and social inequities: A tool for identification of mobility needs and evaluation of transport investments. Journal of Transport Geography, 24, 142-154. https://doi. org/10.1016/j.jtrangeo.2011.12.004

Büscher, M., Sheller, M., \& Tyfield, D. (2016). Mobility intersections: Social research, social futures. Mobilities, 11(4), 485-497. https://doi.org/10.1080/17450101.201 $\underline{6.1211818}$

Cedeus. (2019). Informe Final. Diagnóstico y propuestas participativas para el re-diseño Barrio San Eugenio. Centro de Desarrollo Urbano Sustentable. 
Cox, T. y Hurtubia, R. (2016). Vectores de expansión urbana y su interacción con los patrones socioeconómicos existentes en la ciudad de Santiago. EURE (Santiago), 42(127), 185207. https://doi.org/10.4067/S0250-71612016000300008

Delaunay, D., Fournier, J. M. y Contreras, Y. (2013). ¿ Es posible medir el capital de movilidad para evaluar sus diferenciaciones sociodemográfica e intraurbana? El caso de los habitantes del Área Metropolitana de Santiago de Chile. Estudios demográficos y urbanos, 28(1), 9-51.

Demoraes, F., Contreras, Y. y Piron, M. (2016). Localización residencial, posición socioeconómica, ciclo de vida y espacios de movilidad cotidiana en Santiago de Chile. Revista Transporte y Territorio, 15(15), 274-301. https:// doi.org/10.34096/rtt.i15.2861

Dirección de Estudios Previsionales. (2019). Informe estadístico mensual del Pilar Solidario-Septiembre 2019. Subsecretaría de Previsión Social.

Figueroa Martínez, C. y Forray Claps, R. (2015). Movilidad femenina: los reveses de la utopía socio-espacial en las poblaciones de Santiago de Chile. Revista de Estudios Sociales, (54),52-67, http://dx.doi.org/10.7440/res54.2015.04.

Figueroa Martínez, C., Hodgson, F., Mullen, C., \& Timms, P. (2018). Creating inequality in accessibility: The relationships between public transport and social housing policy in deprived areas of Santiago de Chile. Journal of Transport Geography, 67, 102-109, https:// doi.org/10.1016/j.jtrangeo.2017.09.006

Figueroa Martínez, C. y Waintrub, N. (2015). Movilidad femenina en Santiago de Chile: Reproducción de inequidades en la metrópolis, el barrio y el espacio público. urbe. Revista Brasileira de Gestão Urbana, 7(1), 48-61. https://doi. org/10.1590/2175-3369.007.001.AO03

Fuentes-García, A., Sánchez, H., Lera, L., Cea, X. y Albala, C. (2013). Desigualdades socioeconómicas en el proceso de discapacidad en una cohorte de adultos mayores de Santiago de Chile. Gaceta Sanitaria, 27(3), 226-232. http://dx.doi.org/10.1016/j.gaceta.2012.11.005
Garretón, M. (2012). Desigualdad espacial y utilidad social: Esfuerzos de movilidad y accesibilidad en el Gran Santiago. Territorios, 2(25), 36-64.

Goins, R. T., Jones, J., Schure, M., Rosenberg, D. E., Phelan, E. A., Dodson, S., \& Jones, D. L. (2015). Older Adults' Perceptions of Mobility: A Metasynthesis of Qualitative Studies. The Gerontologist, 55(6), 929-942. https://doi. org/10.1093/geront/gnu014

Gajardo, J., Navarrete, E., López, C., Rodríguez, J., Rojas, A., Troncoso, S. y Rojas, A. (2012). Percepciones de personas mayores sobre su desempeño en el uso de transporte público en Santiago de Chile. Revista Chilena de Terapia Ocupacional, 12(1), 88-102. https://doi.org/10.5354/0719$\underline{5346.2012 .22055}$

Gutierrez, F. J., \& Ochoa, S. F. (2016). Mom, I Do Have a Family!: Attitudes, Agreements, and Expectations on the Interaction with Chilean Older Adults. In Proceedings of the 19th ACM Conference on Computer-Supported Cooperative Work \& Social Computing, pp. 1402-1411. https://doi.org/10.1145/2818048.2820000

Herrmann-Lunecke, M. G., Mora, R., \& Sagaris, L. (2020). Persistence of walking in Chile: lessons for urban sustainability. Transport reviews, 40(2), 135-159, https:// doi.org/10.1080/01441647.2020.1712494

Iglesias, V., Giraldez, F., Tiznado-Aitken, I., \& Muñoz, J. C. (2019). How Uneven is the Urban Mobility Playing Field? Inequalities among Socioeconomic Groups in Santiago De Chile. Transportation Research Record, 2673(11), 5970. https://doi.org/10.1177/0361198119849588

Imilan, W., Jirón, P. e Iturra, L. (2015). Más allá del barrio: Habitar Santiago en la movilidad cotidiana. Revista Antropologías del Sur, 3, 87-103.

Instituto Nacional de Estadísticas. (2017). Chile: Proyecciones y Estimaciones de Población. Total País. Período de Información: 1950-2050. Instituto Nacional de Estadísticas. 
Jirón, P. (2010). Mobile Borders in Urban Daily Mobility Practices in Santiago de Chile. International Political Sociology, 4, 66-79.

Jirón, P. y Gómez, J. (2018). Interdependencia, cuidado y género desde las estrategias de movilidad en la ciudad de Santiago. Tempo Social, 30(2), 55-72, https://doi. org/10.11606/0103-2070.ts.2018.142245

Jirón, P., Carrasco, J. A., \& Rebolledo, M. (2020). Observing gendered interdependent mobility barriers using an ethnographic and time use approach. Transportation Research Part A: Policy and Practice, 140, 204-214. https:// doi.org/10.1016/j.tra.2020.08.018

Jirón, P., Imilan, W., \& Iturra, L. (2016). Relearning to travel in Santiago: The importance of mobile place-making and travelling know-how. Cultural Geographies, 23(4), 599-614. https://doi.org/10.1177/1474474015622141

Jirón, P. y Mansilla, P. (2014). Las consecuencias del urbanismo fragmentador en la vida cotidiana de habitantes de la ciudad de Santiago de Chile. EURE (Santiago), 40(121), 5-28, https://doi.org/10.4067/S0250-71612014000300001

Jouffe, Y. (2011). Las clases socio-territoriales entre movilidad metropolitana y repliegue barrial. ¿Tienen los pobladores pobres una movilidad urbana de clase? Revista Transporte y Territorio, 4(0), 84-117.

Jouffe, Y. y Lazo Corvalán, A. (2010). Las prácticas cotidianas frente a los dispositivos de la movilidad: Aproximación política a la movilidad cotidiana de las poblaciones pobres periurbanas de Santiago de Chile. EURE, 36(108), 29-47. https://doi.org/10.4067/S0250-71612010000200002

Lazo, A. y Calderón, R. (2014). Los anclajes en la proximidad y la movilidad cotidiana: Retrato de tres barrios de la ciudad de Santiago de Chile. EURE (Santiago), 40(121), 121-140, http://dx.doi.org/10.4067/S0250-71612014000300006
Link, F., Valenzuela, F. y Fuentes, L. (2015). Segregación, estructura y composición social del territorio metropolitano en Santiago de Chile: Complejidades metodológicas en el análisis de la diferenciación social en el espacio. Revista de Geografía Norte Grande, 62, 151168. https://doi.org/10.4067/S0718-34022015000300009

Lucas, K. (2012). Transport and social exclusion: Where are we now? Transport Policy, 20, 105-113. https://doi. org/10.1016/j.tranpol.2012.01.013

Lucas, K., Mattioli, G., Verlinghieri, E., \& Guzman, A. (2016). Transport Poverty and Its Adverse Social Consequences. Proceedings of the Institution of Civil Engineers - Transport, 169(6), 353-365.

Lucas, K., Wee, B. van, \& Maat, K. (2016). A method to evaluate equitable accessibility: Combining ethical theories and accessibility-based approaches. Transportation, 43(3), 473-490. https://doi.org/10.1007/s11116-015-9585-2

Martens, K. (2006). Basing Transport Planning on Principles of Social Justice. Berkeley Planning Journal, 19, 1-17. https://doi.org/10.5070/BP319111486

Martens, K. (2016). Transport justice: Designing fair transportation systems. London: Routledge.

Martens, K. (2018). Ageing, impairments and travel: Priority setting for an inclusive transport system. Transport Policy, 63, 122130. https://doi.org/10.1016/J.TRANPOL.2017.12.001

Meijering, L., Hoven, B. van, \& Yousefzadeh, S. (2019). "I think I'm better at it myself": The Capability Approach and Being Independent in Later Life. ResearchonAgeing and Social Policy, 7(1), 229-259. https://doi.org/10.17583/rasp.2019.3678

Niehaus, M., Galilea, P., \& Hurtubia, R. (2016). Accessibility and equity: An approach for wider transport project assessment in Chile. Research in Transportation Economics, 59, 412-422. https://doi.org/10.1016/j.retrec.2016.05.003 
Nordbakke, S. (2013). Capabilities for mobility among urban older women: Barriers, strategies and options. Journal of Transport Geography, 26, 166-174. https:// doi.org/10.1016/j.jtrangeo.2012.10.003

Nordbakke, S., \& Schwanen, T. (2014). Well-being and Mobility: A Theoretical Framework and Literature Review Focusing on Older People. Mobilities, 9(1), 104-119. https://doi. org/10.1080/17450101.2013.784542

Olivi, A., Fadda, G. y Reyes, V. (2016). Movilidad urbana y calidad de vida de las personas mayores en una ciudad vertical. El caso de Valparaíso, Chile. Revista Márgenes Espacio Arte y Sociedad, 13(19), 38-47.

Pucci, P., \& Vecchio, G. (2019a). Enabling Mobilities. Planning Tools for People and Their Mobilities. Springer.

Pucci, P., \& Vecchio, G. (2019b). Trespassing for mobilities. Operational directions for addressing mobile lives. Journal of Transport Geography, 81. https://doi.org/10.1016/j. jtrangeo.2019.102536

Ryan, J., Wretstrand, A., \& Schmidt, S. M. (2019). Disparities in mobility among older people: Findings from a capabilitybased travel survey. Transport Policy, 79, 177-192. https:// doi.org/10.1016/j.tranpol.2019.04.016

Sánchez-González, D., \& Rodríguez-Rodríguez, V. (2016). Environmental Gerontology in Europe and Latin America. Policies and Perspectives on Environment and Aging. Springer.

Schwanen, T. (2018). Towards decolonised knowledge about transport. Palgrave Communications, 4(1), 79. https:// doi.org/10.1057/s41599-018-0130-8

Schwanen, T., Banister, D., \& Bowling, A. (2012). Independence and mobility in later life. Geoforum, 43(6), 1313-1322. https://doi.org/10.1016/j.geoforum.2012.04.001
Sheller, M., \& Urry, J. (2006). The new mobilities paradigm. Environment and Planning A: Economy and Space, 38(2), 207-226. https://doi.org/10.1068/a37268

Steiniger, S., Fuentes, C., Villegas, R., Ardiles, R., Rojas, C. y Poorazizi, E. (2018). AccesoBarrio - Medición de la accesibilidad urbana usando perfiles demográficos y OpenStreetMap. Ingeniería de Transporte, 21(1). https:// estudiosdetransporte.org/sochitran/issue/view/28

Stjernborg, V., Wretstrand, A., \& Tesfahuney, M. (2015). Everyday Life Mobilities of Older Persons - A Case Study of Ageing in a Suburban Landscape in Sweden. Mobilities, 10(3), 383-401. https://doi.org/10.1080/174 50101.2013.874836

Tiznado-Aitken, I., Muñoz, J. C., \& Hurtubia, R. (2018). The Role of Accessibility to Public Transport and Quality of Walking Environment on Urban Equity: The Case of Santiago de Chile. Transportation Research Record, 2672(35), 129-138. https://doi.org/10.1177/0361198118782036

Umstattd Meyer, M. R., Janke, M. C., \& Beaujean, A. A. (2014). Predictors of Older Adults' Personal and Community Mobility: Using a Comprehensive Theoretical Mobility Framework. The Gerontologist, 54(3), 398-408. https:// doi.org/10.1093/geront/gnt054

United Nations (2017). World Population Prospects: The 2017 Revision, Volume II: Demographic Profiles. New York: United Nations.

Universidad Alberto Hurtado, Observatorio Social. (2014). Actualización y recolección de información del sistema de transporte urbano, IX Etapa: Encuesta Origen Destino Santiago 2012. Encuesta origen destino de viajes 2012 (Documento Difusión). Sectra.

Ureta, S. (2015). Assembling Policy. Transantiago, Human Devices, and the Dream of a World-Class Society. Boston: MIT Press. 
Wee, B. van. (2011). Transport and Ethics: Ethics and the Evaluation of Transport Policies and Projects. Elgar.

Vecchio, G. (2020). Microstories of everyday mobilities and opportunities in Bogotá: A tool for bringing capabilities into urban mobility planning. Journal of Transport Geography, 83. https://doi.org/10.1016/j.jtrangeo.2020.102652

Vecchio, G., Castillo, B., Steiniger, S., \& Muñoz, J. C. (2019). Transporte y adultos mayores: ¿a un 'metro' de la equidad? Cedeus - Centro de Desarrollo Urbano Sustentable.

Vecchio, G., Tiznado-Aitken, I., \& Hurtubia, R. (2020). Transport and equity in Latin America: a critical review of socially oriented accessibility assessments. Transport Reviews, 40(3), 354-381. https://doi.org/10.1080/01441647.202 0.1711828
Wallace, S. P., \& Gutiérrez, V. F. (2005). Equity of access to health care for older adults in four major Latin American cities. Revista Panamericana de Salud Pública, 17(5-6), 394-409. https://doi.org/10.1590/s1020-49892005000500012

Webber, S. C., Porter, M. M., \& Menec, V. H. (2010). Mobility in Older Adults: A Comprehensive Framework. The Gerontologist, 50(4), 443-450. https://doi.org/10.1093/ geront/gnq013 